\title{
New planetary nebulae towards the galactic center ${ }^{\star}$
}

\author{
E. Cappellaro, F. Sabbadin, S. Benetti, and M. Turatto \\ Osservatorio Astronomico di Padova, vicolo dell'Osservatorio 5, 35122 Padova, Italy \\ Received 28 June 2001 / Accepted 3 August 2001

\begin{abstract}
Sixteen planetary nebulae and a shocked peculiar nebula were discovered in the direction of the Galactic Center by means of direct inspection of the Palomar Observatory and ESO/SERC sky surveys. For all objects we obtained narrow band $\mathrm{H} \alpha$ imaging and medium resolution spectroscopy, allowing us to derive the basic parameters of the emission nebulae. For half of the nebulae of the sample, the central star candidate was identified and an estimate of the star temperature and luminosity is given.
\end{abstract}

Key words. ISM: planetary nebulae: general - ISM: general

\section{Introduction}

The late evolution of intermediate and low mass stars is characterized by the "planetary nebula" (PN) phase. Implicit in this sentence is the importance of $\mathrm{PNe}$ in all problems involving stellar evolution and galactic population, interstellar medium kinematics and chemical enrichment.

Two decades ago, when the number of identified PNe was about 1100, Wyatt (1978) stated: "Perhaps we should have said for the total number of Planetary Nebulae in the Galaxy 20000 or 30000 plus or minus 25000 !". Today we know almost 2000 galactic PNe (Acker et al. 1992; Kohoutek 1994, 1997), but the foregoing sentence is still valid (Pottasch 1996). We have reached the apparent paradox that the space density, distribution and population of $\mathrm{PNe}$ is almost better defined in some external galaxies - such as NGC 224, NGC 4486 and NGC 4594 than in the Galaxy (Jacoby 1997; Ciardullo et al. 1998). This situation, only partially due to observational biases (i.e. strong interstellar absorption in the direction of the Galactic Center), is revealing of the emphasis of current astronomical research on extragalactic targets.

This explains why it is important to:

a) increase the number of galactic $\mathrm{PNe}$ (mainly in the Galactic Center region);

b) study in detail the new, faint, distant PNe to determine the main parameters (morphology, kinematics, distance, physical conditions, ionic and total abundances of the gas and spectral type, temperature and luminosity of the central star);

Send offprint requests to: E. Cappellaro,

e-mail: cappellaro@pd.astro.it

* Figures 1a to $1 \mathrm{e}$ are only available in electronic form at http://www . edpsciences.org c) analyze in the galactic context the statistical properties of this class of objects.

This paper addresses points a) and (partly) b).

\section{Search for new PNe}

Nowadays, the discovery of galactic PNe is performed by means of:

1. direct inspection of the Palomar Observatory and ESO/SERC sky survey plates (Weinberger 1977; Weinberger et al. 1983; Kerber et al. 1996);

2. radio and/or optical identification of IRAS point sources presenting characteristic infrared colors (Pottasch et al. 1988; Van de Steene \& Pottasch 1993; Kistiakowsky \& Helfand 1995);

3. comparison of deep $\mathrm{H} \alpha$-on and $\mathrm{H} \alpha$-off imaging (Beaulieu et al. 1999);

4. objective-prism spectra (Kohoutek 1994);

5. red-infrared comparison (Sabbadin 1986; Cappellaro et al. 1994).

Since the late 80 s, we have largely used methods 1) and 5) to detect a huge number of candidates; the nature of some of them has been confirmed through detailed follow-up spectroscopy (Turatto et al. 1990; Cappellaro et al. 1990, 1994).

Here we report the identification and spectroscopic confirmation of $16 \mathrm{PNe}$ and 1 peculiar nebula, discovered by direct inspection (method 1) of eight sky survey plates corresponding to an area of about 240 square degrees. They were already included in a preliminary list of candidate PNe by Zanin et al. (1997).

The standard designations and coordinates of these new objects are listed in Table 1. 
Table 1. List of new PNe.

\begin{tabular}{|c|c|c|c|c|c|c|c|c|}
\hline $\begin{array}{l}\text { Designation } \\
\text { PN G }\end{array}$ & \multicolumn{2}{|c|}{$(2000.0)$} & $\begin{array}{l}\text { Instrument } \\
\text { Imaging }\end{array}$ & $\begin{array}{l}\text { seeing } \\
{[\text { arcsec] }}\end{array}$ & $\begin{array}{l}\text { Instrument } \\
\text { Spectroscopy }\end{array}$ & $\begin{array}{c}\text { wavelength } \\
\text { range }[\AA]\end{array}$ & $\begin{array}{l}\text { res. } \\
{[\AA]}\end{array}$ & ref. \\
\hline $354.4+03.2$ & 171901.8 & -314741 & Dan1.5 & 1.2 & $\mathrm{ESO} 1.5+\mathrm{B} \& \mathrm{C}$ & $3600-9400$ & 6 & \\
\hline $354.5-03.9$ & $1748 \quad 16.3$ & -353828 & Dan1.5 & 1.3 & ESO3.6+EF & $3700-6850$ & 14 & \\
\hline $355.1+04.7$ & 171503.1 & -302038 & Dan1.5 & 1.2 & $\mathrm{ESO} 1.5+\mathrm{B} \& \mathrm{C}$ & 3600-9400 & 6 & $1,2,3$ \\
\hline $356.4-02.5^{a}$ & $1747 \quad 17.8$ & -331539 & Dan1.5 & 1.9 & & & & 2,4 \\
\hline $356.5+02.2$ & 172807.9 & -303818 & Dan1.5 & 1.2 & $\mathrm{ESO} 1.5+\mathrm{B} \& \mathrm{C}$ & $3800-9400$ & 6 & \\
\hline $357.5-02.4$ & 174937.9 & -321628 & Dan1.5 & 1.6 & $\mathrm{ESO} 1.5+\mathrm{B} \& \mathrm{C}$ & 3600-9400 & 6 & \\
\hline $000.5-05.3$ & 180834.7 & -310652 & ESO3.6 & 1.2 & $\mathrm{ESO} 1.5+\mathrm{B} \& \mathrm{C}$ & 3800-9400 & 6 & 5 \\
\hline $000.7-06.1$ & 181214.4 & -311959 & Dan1.5 & 1.7 & & & & 3,5 \\
\hline $001.3+06.3$ & 172453.8 & -241926 & Dan1.5 & 1.9 & $\mathrm{ESO} 1.5+\mathrm{B} \& \mathrm{C}$ & $3700-7600$ & 4 & \\
\hline $002.0+06.6$ & 172541.8 & -233832 & Dan1.5 & 2.2 & ESO3.6+EF & $3700-6850$ & 14 & \\
\hline $002.2-01.2$ & 175545.5 & -273947 & Dan1.5 & 13 & $\mathrm{ESO} 1.5+\mathrm{B} \& \mathrm{C}$ & $3600-9400$ & 6 & \\
\hline $008.8+03.8$ & 175108.7 & -192548 & Dan1.5 & 1.2 & $\mathrm{ESO} 1.5+\mathrm{B} \& \mathrm{C}$ & 3800-9500 & 6 & \\
\hline $009.9+04.5$ & 175047.3 & -180330 & Dan1.5 & 1.4 & ESO3.6+EF & $3700-6850$ & 14 & \\
\hline $011.2-02.7$ & 182035.7 & -203029 & Dutch & 1.5 & $\mathrm{ESO} 1.5+\mathrm{B} \& \mathrm{C}$ & 3200-9900 & 12 & \\
\hline $011.7+00.2^{b}$ & 181019.9 & -183910 & Dan1.5 & 1.7 & $\mathrm{ESO} 1.5+\mathrm{B} \& \mathrm{C}$ & $3700-7600$ & 5 & 6,7 \\
\hline $012.2-02.2$ & 182017.5 & -192640 & $\mathrm{ESO} 2.2$ & 1.7 & $\mathrm{ESO} 1.5+\mathrm{B} \& \mathrm{C}$ & $3800-9500$ & 6 & \\
\hline $012.5+04.3$ & 175710.6 & -155618 & Dan1.5 & 2.3 & $\mathrm{ESO} 1.5+\mathrm{B} \& \mathrm{C}$ & 3800-9500 & 6 & \\
\hline
\end{tabular}

a) Same as PN K 6-12.

$\left.{ }^{b}\right)$ Same as GPSR5 11.725+0.264.

1 - Cuisinier et al. (1994); 2 - Kohoutek (1997); 3 - Durand et al. (1998); 4 - Kohoutek (1994); 5 - Beaulieu et al. (1999); 6 - Becker et al. (1994); 7- Ratag \& Pottasch (1991).

\section{Observations}

Imaging for most objects was obtained with the CCD camera at the ESO Danish $1.5 \mathrm{~m}$ telescope during four nights: May 4-5 and July 6-7 1993. The detector was the $1024 \times 1024$ pixels CCD TeK ESO\#28 (pixel size $24 \mu \mathrm{m}$, scale $0.38 \mathrm{arcsec} / \mathrm{pix})$. For each candidate we obtained imaging through Johnson-Cousin $B, V, R$ filters (exposure time of a few minutes) and through a narrow $\mathrm{H} \alpha$ interference filter (central wavelength $6562 \AA, F W H M=$ $60 \AA$; exposure time of $20-30 \mathrm{~min}$ ). For the photometric calibration of the broad band filters we observed some photometric standard fields from the list of Landolt (1992) and for the calibration of narrow band imaging we observed through the $\mathrm{H} \alpha$ filter a few spectrophotometric standards from the list of Hamuy et al. (1992, 1994). Additional imaging (cf. Table 1) was obtained on Aug. 24, 1993 with the ESO $3.6 \mathrm{~m}+$ EFOSC (CCD TeK ESO\#26, scale 0.61 arcsec/pix), on July 26, 1992 with the ESO/MPI $2.2 \mathrm{~m}+$ EFOSC2 (CCD Thompson ESO\#19, scale $0.33 \mathrm{arcsec} / \mathrm{pix}$ ) and on Sept. 11, 1993 with the Dutch $0.90 \mathrm{~m}+$ CCD camera (CCD TeK ESO\#29, scale $0.44 \mathrm{arcsec} / \mathrm{pix}$ ). For each object the seeing ( FWHM) measured on the $\mathrm{H} \alpha$ image is reported in Col. 5 of Table 1.

All frames were corrected for bias and flat-fielding using standard IRAF packages. $\mathrm{H} \alpha$ and $R$ band images are presented in the left and middle columns of Fig. 1, respectively. When identified (see next section), the central star candidate(s) is (are) indicated in the $R$ image. The $R$ band frames were subtracted from the $\mathrm{H} \alpha$ frames to obtain the net $\mathrm{H} \alpha$ emission. To this purpose, $R$ band and $\mathrm{H} \alpha$ frames were geometrically and photometrically registered and, if required, the PSF was matched by degradating the image with the better seeing. The resulting image was used a) to obtain the contour maps shown in Fig. 1 (right column) and b) to measure the nebula integrated $\mathrm{H} \alpha$ fluxes (Col. 2 of Table 2), using the IRAF task polyphot. In three cases, indicated by "\#", the absolute calibration of the H $\alpha$ imaging was derived from the flux calibrated spectrum after scaling for the portion of the nebula covered by the slit.

Spectroscopy was obtained with the B\&C spectrograph attached at the ESO $1.5 \mathrm{~m}$ telescope. The detector was the CCD Ford ESO\#24 with $15 \mu \mathrm{m}$ pixel and a spatial scale of $0.82 \mathrm{arcsec} / \mathrm{pix}$. On July 5, 1993 we used the grating \#23 which, with a 2 arcsec wide slit, giving a resolution of 4-5 A. Instead, on July 8 and Aug. 8, 1993 we used the grating \#25 (also with a 2 arcsec slit) obtaining a resolution of $6 \AA$. Typical exposure time was $1 \mathrm{~h}$. Spectra of three objects were obtained on May 16, 1993 with the ESO $3.6 \mathrm{~m}+$ EFOSC and the B300 grism. Exposure time was $20 \mathrm{~min}$. The spectrum of PN G011.2-02.7 was obtained on June 10, 1996 using ESO $1.5 \mathrm{~m}+\mathrm{B} \& \mathrm{C}$ grating \#2 (resolution $12 \AA$ ).

In all cases, for wavelength calibration we secured spectra of an He-Ar lamp and for flux calibration we observed standard stars from the list of Hamuy et al. (1992, 1994). The bidimensional frames were calibrated using the tasks in the IRAF longslit package. After examination of the calibrated frames it turned out that in most cases the $\mathrm{S} / \mathrm{N}$ of the observations was not good enough for investigating spatial variations of the emission line intensities. Therefore, the spectra were integrated along the slit and the line intensities measured on the resulting tracing. 
The relative intensities $(\mathrm{H} \beta=100)$ of the detected lines are listed in Table 4. The estimated error ranges from $5 \%$ for brightest lines to $20-30 \%$ for the faintest ones. The measurements marked with a colon have error larger than $50 \%$, due to some specific problems (e.g. cosmic ray contamination, uncertainty in the flux calibration at the ends of the useful wavelength range, etc.). Because of the low resolution, some of the lines (in particular $\mathrm{H} \alpha+[\mathrm{NII}]$ and the [SII] doublet) appear blended in the spectra obtained with EFOSC at the ESO $3.6 \mathrm{~m}$ telescope In these cases, the intensities of the individual lines were measured via a multi-Gaussian fit (this is indicated by "** in Table 4).

Two objects for which we could not obtain spectroscopic observations (PN G356.4-02.5 and G000.7-06.1), were meanwhile detected and spectroscopic confirmed by other authors (cf. Col. 9 of Table 1).

\section{Results}

The relative emission line intensities confirm that all but one (PN G011.2-02.7, cf. Sect. 5) of the new candidates are photoionized nebulae. Indeed, by plotting the objects in the diagnostic diagram $\log (\mathrm{H} \alpha /[\mathrm{NII}])$ vs. $\log (\mathrm{H} \alpha /[\mathrm{SII}])$ (Tajitsu et al. 1999), they nicely spread along the strip occupied by PNe.

The interstellar extinction, $c(\mathrm{H} \beta)$, was derived from the observed $\mathrm{H} \alpha / \mathrm{H} \beta$ ratio, adopting 2.85 for the intrinsic value (case $\mathrm{B}$ recombination, temperature $10^{4} \mathrm{~K}$, electron density $10^{4} \mathrm{~cm}^{-3}$; Brocklehurst 1971) and the standard extinction law (Seaton 1979). Thus:

$c(\mathrm{H} \beta)=\frac{1}{0.325} \log \frac{F(\mathrm{H} \alpha) / F(\mathrm{H} \beta)}{2.85}$.

The values of $c(\mathrm{H} \beta)$ for all objects are listed in Col. 4 of Table 2.

The excitation class of the nebulae (Col. 5 of Table 2) has been computed using the prescription of Dopita \& Meatheringham (1990), namely:

E.C. $=0.45\left[\frac{F(5007)}{F(\mathrm{H} \beta)}\right] \quad(0.0<$ E.C. $<5.0)$

or

E.C. $=5.54\left[\frac{F(4686)}{F(\mathrm{H} \beta)}+0.78\right] \quad(5.0 \leq$ E.C. $<10.0)$.

The electron density $\mathrm{Ne}$ (Col. 6) was derived from the [SII] 6716/6731 ratio for an assumed electron temperature $T \mathrm{e}=10000 \mathrm{~K}$ (Osterbrock 1989).

We also report an estimate of the heliocentric radial velocities averaged through all the lines detected in each spectrum (Col. 7, in parenthesis is the internal error).

One of the most difficult parameter to measure for $\mathrm{PNe}$ is the distance. In Table 2, we report different estimates based on the statistical methods commonly used in the literature. One estimate, $D_{\text {ext }}$ (Col. 8), combines the measured PNe extinction with the spatial distribution of the dust in the solar neighbours (Lucke 1978). The main uncertainty is that the extinction is patchy and changes significantly even for small variation of the line of sight. In addition, the available maps extend only up to $2 \mathrm{kpc}$ from the Sun, thus the extrapolation to higher distances is quite arbitrary. Given that, the average uncertainties in individual measurements can be as large as $\pm 50 \%$.

Another approach often used to derive the PNe distances is the Shklovsky method, based on the assumption that the ionized nebular mass is constant and the nebula is optically thin. Following Pottasch (1983) and Boffi \& Stanghellini (1994) we compute:

$$
D_{F(\mathrm{H} \beta)}=22.8 \frac{M^{2 / 5}}{F(\mathrm{H} \beta)^{1 / 5}\left(r^{\prime \prime}\right)^{3 / 5}}
$$

where $D_{F(\mathrm{H} \beta)}$ is the distance in kpc, $M$ is the nebular mass in solar units, $r^{\prime \prime}$ is the radius in arcsec, and $F(\mathrm{H} \beta)$ is the integrated $\mathrm{H} \beta$ flux in units $10^{-11} \mathrm{erg} \mathrm{cm}^{-2} \mathrm{~s}^{-1}$. The latter was derived from the measured $F(\mathrm{H} \alpha)$ and $c(\mathrm{H} \beta)$ assuming $T \mathrm{e}=10^{4} \mathrm{~K}$. Using $0.2 M_{\odot}$ for the nebular mass, we can readily derive the distances reported in Col. 9 of Table 2 .

A comparison of Cols. 8 and 9 of Table 2 shows that, considering the large uncertainties, the distances derived with different methods are in fair agreement. The adopted value, reported in Col. 10, is the average of the two determinations and is used to derive the nebular linear radius (Col. 11).

Note that only one of the objects appears to have an IRAS counterpart (PN G011.7+00.2, corresponds to IRAS18073-1839, $F(25 \mu)=1.99 \mathrm{mJy})$. In a previous paper (Cappellaro et al. 1994) we presented a plot of the near infrared emission $F(25 \mu)$ vs. the surface brightness $F(\mathrm{H} \beta) /$ radius $^{2}$ for the known PNe closer than 2 kpc. We verified, after Pottasch (1992), that there is a linear correlation between these two quantities. Since our new PNe have surface brightness $\log F(\mathrm{H} \beta) /$ radius $^{2}<-14.5$, their expected $F(25 \mu)$ flux at a distance of $1 \mathrm{kpc}$ is $<0.5 \mathrm{Jy}$. Being the new PNe more distant than $1 \mathrm{kpc}$ and given that close to the Galactic plane the signal-to-noise cut-off for inclusion in the IRAS PSC is $0.8 \mathrm{Jy}$, their non-detection by IRAS is not surprising.

The central star candidate was identified for half of the nebulae, based on the location close to the geometrical center of the nebula and on the relatively blue color. They are listed in Table 3 along with the measured magnitudes (Cols. 2-4). We use these data and standard prescriptions (Pottasch 1983; Aller 1984; Osterbrock 1989) to derive the HI and HeII Zanstra temperatures (Cols. 5 and 6). Finally, from $T_{\mathrm{Z}}(\mathrm{HI})$ and $T_{\mathrm{Z}}(\mathrm{HII})$ and the bolometric correction given by Schönberner (1981), we derive an estimate of the central star luminosity (Cols. 6 and 7).

It is known that there is only a week correlation between Zanstra temperatures and excitation classes (Gleizes et al. 1989). In our sample, this is almost completely washed out by the uncertainties in the Zanstra temperature determinations. Yet, of the alternative central star candidates for PNG 009.9+04.5, because of its high excitation class, we believe that the more plausible 
Table 2. Integrated properties.

\begin{tabular}{|c|c|c|c|c|c|c|c|c|c|c|}
\hline PN G & $\begin{array}{c}F(\mathrm{H} \alpha) \\
{\left[10^{-13} \mathrm{erg} \mathrm{s}^{-1} \mathrm{~cm}^{-2}\right]}\end{array}$ & $\begin{array}{c}\text { size } \\
{[\operatorname{arcsec}]}\end{array}$ & $c(\mathrm{H} \beta)$ & E.C. & $\begin{array}{r}N e \\
{\left[\mathrm{~cm}^{-3}\right]}\end{array}$ & $\begin{array}{r}v_{\mathrm{Hel}} \\
{\left[\mathrm{km} \mathrm{s}^{-1}\right]}\end{array}$ & $\begin{array}{l}D_{\text {ext. }} \\
{[\mathrm{kpc}]}\end{array}$ & $\begin{array}{r}D_{F(\mathrm{H} \beta)} \\
{[\mathrm{kpc}]}\end{array}$ & $\begin{array}{r}D_{\text {adop }} \\
{[\mathrm{kpc}]}\end{array}$ & $\begin{array}{r}R \\
{[\mathrm{pc}]}\end{array}$ \\
\hline $354.4+03.2$ & 1.3 & 11 & 1.58 & 7 & 1450 & $50(21)$ & 5.3 & 6.7 & 6.0 & 0.16 \\
\hline $354.5-03.9$ & 1.8 & $77 \times 42$ & 0.77 & 3 & 600 & $-75(24)$ & 2.6 & 3.8 & 3.0 & 0.43 \\
\hline $355.1+04.7$ & 3.8 & $46 \times 34$ & 1.40 & 4 & 230 & $112(28)$ & 4.7 & 3.5 & 4.0 & 0.38 \\
\hline $356.4-02.5$ & 1.5 & 14 & & & & & - & - & - & - \\
\hline $356.5+02.2$ & 0.4 & $17 \times 15$ & $>2.29$ & 3 & 650 & -175 (13) 0 & $>7.7$ & 5.3 & 6.0: & 0.23 : \\
\hline $357.5-02.4$ & 0.9 & $9 \times 6$ & 2.53: & 2 & 1250 & $-203(14)$ & 8.5 & 7.2 & 8.0 & 0.14 \\
\hline $000.5-05.3$ & $10.5^{\#}$ & 23 & 0.81 & 2 & 70 & $19(7)$ & 2.7 & 4.9 & 3.5 & 0.19 \\
\hline $000.7-06.1$ & 11.7 & $48 \times 29$ & & & & & - & - & - & - \\
\hline $001.3+06.3$ & 31.0 & $55 \times 37$ & $>0.50$ & 4 & 180 & 87 (8) 0 & $>0.8$ & 1.6 & 1.5 & 0.17 \\
\hline $002.0+06.6$ & 2.2 & 19 & 1.80 & 0 & 250 & $95(23)$ & 3.0 & 5.0 & 4.0 & 0.19 \\
\hline $002.2-01.2$ & 1.2 & $11 \times 8$ & 3.55 & 4 & 1300 & $106(21)$ & 6.0 & 3.2 & 4.5 & 0.10 \\
\hline $008.8+03.8$ & 4.0 & 11 & 1.77 & 4 & & $225(33)$ & 3.0 & 5.5 & 4.0 & 0.11 \\
\hline $009.9+04.5$ & 2.4 & $35 \times 26$ & 1.22 & 8 & 120 & 49 (19) & 2.1 & 5.1 & 3.5 & 0.25 \\
\hline $011.2-02.7$ & $1.6^{\#}$ & 38 & 1.92 & 2 & 520 & $-12(20)$ & 3.2 & 3.4 & $3.3^{*}$ & 0.30 \\
\hline $011.7+00.2$ & 1.0 & $17 \times 12$ & $>2.60$ & 2 & 1950 & $-20(33) 0$ & $>4.3$ & 4.5 & 4.5 & 0.32 \\
\hline $012.2-02.2$ & $0.7^{\#}$ & 10 & 2.17 & 3 & & $-23(25)$ & 3.7 & 7.2 & 5.0 & 0.12 \\
\hline $012.5+04.3$ & 3.9 & 32 & 2.46 & 4 & 270 & $50(21)$ & 4.2 & 2.2 & 3.2 & 0.25 \\
\hline
\end{tabular}

\# From spectrum.

- See comments in Sect. 5.

Table 3. Central stars.

\begin{tabular}{|c|c|c|c|c|c|c|c|}
\hline PN & $B$ & $V$ & $R$ & $\log T_{\mathrm{Z}}(\mathrm{HI})$ & $\log T_{\mathrm{Z}}(\mathrm{HeII})$ & $\log \frac{L}{L_{\odot}}(\mathrm{HI})$ & $\log \frac{L}{L \odot}(\mathrm{HeII})$ \\
\hline $354.4+03.2$ & 22.85 & 21.62 & 21.14 & 4.74 & 4.96 & 2.1 & 2.6 \\
\hline $354.5-03.9$ & 19.08 & 18.34 & 17.90 & 4.53 & 4.85 & 1.4 & 2.3 \\
\hline $355.1+04.7$ & 21.78 & 20.45 & 19.81 & 4.75 & & 2.5 & \\
\hline $356.5+02.2$ & $>22.8$ & 22.36 & 21.51 & $>5.2$ & & $>3.5$ & \\
\hline $000.5-05.3$ & - & - & 19.17 & 4.65: & 4.94 & 1.4: & 2.3: \\
\hline $000.7-06.1$ & 18.94 & 18.49 & 18.45 & & & & \\
\hline $001.3+06.3$ & 19.52 & 18.16 & 17.39 & 4.80 & & $>1.5$ & \\
\hline $002.0+06.6$ & $>20.0$ & 19.54 & 18.80 & 4.57 & & 2.2 & \\
\hline $009.9+04.5-\mathrm{a}$ & $>20.0$ & 18.66 & 17.65 & 4.53 & 4.85 & 2.1 & 2.6 \\
\hline $009.9+04.5-b$ & & $>20.0$ & 20.12 & 4.75: & 4.99: & 1.6: & 2.3: \\
\hline $012.5+04.3-\mathrm{a}$ & $>18.5$ & 18.51 & 18.69 & 4.60 & 4.87 & 2.5 & 3.3 \\
\hline $012.5+04.3-b$ & & 21.6: & 19.63 & 4.88: & 5.01: & 2.4: & 3.0: \\
\hline
\end{tabular}

is the fainter one (b), which shows a higher Zanstra temperature.

\section{Notes on individual objects}

PNG354.4+03.2 A faint, roundish, homogeneous, high ionization disk presenting two bright, quasi-stellar blobs in $\mathrm{PA}=200^{\circ}$. Low ionization emissions, like [NII] and [SII], are present only in these condensations $(\mathrm{H} \alpha / 6584 \AA=7, \mathrm{H} \alpha / 6717+6731 \AA=18)$. The [SII] ratio indicates an electron density $N$ eblobs $_{\text {bl }} \sim 2000 \mathrm{~cm}^{-3}$ and the $\mathrm{H} \alpha$ surface brightness ratio for blobs/disk suggests that $N$ eblobs $_{\text {blo }}=1.5 \mathrm{Ne}_{\text {disk }}$.

PNG355.1+04.7 A bipolar, stratified PN independently discovered by Terzan and studied by Cuisinier et al. (1994). They obtain $\log F(\mathrm{H} \beta)=-12.4 \mathrm{erg} \mathrm{cm}^{-2} \mathrm{~s}^{-1}$, $v_{\text {hel }}=64 \pm 30 \mathrm{~km} \mathrm{~s}^{-1}, c(\mathrm{H} \beta)=0.80:, N \mathrm{e}=300 \mathrm{~cm}^{-3}$ and distance $<3.5 \mathrm{kpc}$, to be compared with our values: $\log F(\mathrm{H} \beta)=-13.33 \mathrm{erg} \mathrm{cm}^{-2} \mathrm{~s}^{-1}, v_{\text {hel }}=112 \pm 29 \mathrm{~km} \mathrm{~s}^{-1}$, $N \mathrm{e}=230$ and distance $=3.5 \mathrm{kpc}$.

PNG356.4-02.5 A faint, bipolar disk located in a heavily obscured galactic region. Independently discovered by Kohoutek (1994) on ESO objective-prism plates.

PNG356.5 +02.2 An inhomogeneous ring presenting large stratification effects, projected in a very absorbed field, probably excited by a very hot central star.

PNG000.5-05.3 A roundish, quite homogeneous, little absorbed, mean excitation disk independently discovered by Beaulieu et al. (1999) on $\mathrm{H} \alpha$-on and $\mathrm{H} \alpha$-off frames taken with the $1.0 \mathrm{~m}$ telescope at Mount Stromlo and Siding Spring Observatories. They derive $\log F(\mathrm{H} \alpha)=$ $-11.88 \mathrm{erg} \mathrm{cm}^{-2} \mathrm{~s}^{-1}$ and $v_{\text {hel }}=3 \pm 11 \mathrm{~km} \mathrm{~s}^{-1}$, to be compared with our values: $\log F(\mathrm{H} \alpha)=-11.98 \mathrm{erg} \mathrm{cm}^{-2} \mathrm{~s}^{-1}$ and $v_{\text {hel }}=19 \pm 7 \mathrm{~km} \mathrm{~s}^{-1}$. 


\begin{tabular}{|c|c|c|c|c|c|c|c|c|c|c|c|c|c|c|c|}
\hline line & $\begin{array}{r}354.4 \\
+03.2\end{array}$ & $\begin{array}{r}354.5 \\
-03.9\end{array}$ & $\begin{array}{r}355.1 \\
+04.7\end{array}$ & $\begin{array}{r}356.5 \\
+02.2\end{array}$ & $\begin{array}{r}357.5 \\
-02.4\end{array}$ & $\begin{array}{r}000.5 \\
-05.3\end{array}$ & $\begin{array}{r}001.3 \\
+06.3\end{array}$ & $\begin{array}{r}002.0 \\
+06.6\end{array}$ & $\begin{array}{r}002.2 \\
-01.2\end{array}$ & $\begin{array}{r}008.8 \\
+03.8\end{array}$ & $\begin{array}{r}009.9 \\
+04.5\end{array}$ & $\begin{array}{c}011.2 \\
-02.7\end{array}$ & $\begin{array}{r}011.7 \\
+00.2\end{array}$ & $\begin{array}{r}012.2 \\
-02.2\end{array}$ & $\begin{array}{r}012.5 \\
+04.3\end{array}$ \\
\hline [OII] 3726-29 & & 80 & & & & & & & & & 100 & 646 & & & \\
\hline [NeIII] 3869 & & 39 & & & & 31 & & & & & 63 & & & & \\
\hline $\mathrm{H} \delta$ & 22 & 19 & & & & 23 & & & & & & & & & \\
\hline $\mathrm{H} \gamma$ & 28 & 37 & & & & 44 & & 33 & & & 36 & & & & \\
\hline [OIII] 4363 & & & & & & & & & & & 15 & & & & \\
\hline HeII 4686 & 53 & 25 & & & & 20 & & & & 72 & 68 & & & 40: & 36: \\
\hline $\mathrm{H} \beta$ & 100 & 100 & 100 & $100^{\#}$ & 100: & 100 & 100: & 100 & 100: & 100 & 100 & 100 & $100^{\#}$ & 100 & 100 \\
\hline [OIII] 4959 & 363 & 235 & 266 & 190 & 150: & 171 & & 8 & 307 & 264 & 513 & 150: & 132 & 231 & 270 \\
\hline [OIII] 5007 & 1144 & 683 & 830 & 568 & 435 & 538 & 180: & 25 & 932 & 814 & 1562 & 450 & 380 & 703 & 840 \\
\hline HeII 5411 & 12 & & & & & & & & & 17 & 8 & & & & \\
\hline [NII] 5755 & & & & 9 & 96: & & & & & & & & & & \\
\hline HeI 5876 & 11 & 12 & 53 & & & 27 & & 45 & & 26 & 20 & & & 40 & \\
\hline [OI] 6300 & & & & & & & 72 & & & & & 202 & & & \\
\hline [NII] 6548 & 27 & $118^{*}$ & 309 & 496 & 199 & 65 & 385 & 237 & 2702 & & $456^{*}$ & $400^{*}$ & 463 & & 790 \\
\hline $\mathrm{H} \alpha$ & 930 & $507^{*}$ & 815 & 1589 & 1895 & 524 & 414 & 1093 & 4054 & 1069 & $711^{*}$ & $1200^{*}$ & 1989 & 1443 & 1790 \\
\hline [NII] 6583 & 77 & $352^{*}$ & 942 & 1510 & 626 & 188 & 1204 & 675 & 8378 & $28:$ & $1400^{*}$ & $1256^{*}$ & 1339 & 97 & 2449 \\
\hline HeI 6678 & 11 & 7 & & & & 10 & 27 & 22 & 94 & & & & & & 59 \\
\hline [SII] 6717 & 15 & $55^{*}$ & 133 & 161 & $65:$ & 31 & 161 & $68:$ & 621 & & $210^{*}$ & $533^{*}$ & 112 & & 244 \\
\hline [SII] 6731 & 19 & $55^{*}$ & 110 & 164 & 79: & 23 & 129 & 57: & 763 & & $162^{*}$ & $513^{*}$ & 154 & & 207 \\
\hline$[\mathrm{ArV}] 7005$ & 11 & & & & & & & & & & & & & & \\
\hline HeI 7065 & 8 & & & & & & & & 141 & & & & & & \\
\hline [ArIII] 7135 & 48 & & 86 & 310 & 231 & 28 & & & 979 & 76 & & 73 & 373 & & 206 \\
\hline [OII] 7320-30 & 15 & & & & & & & & 228 & & & 290 & & & \\
\hline [ArIII] 7751 & 22 & & & & 116 & 9 & & & 471 & 28: & & & 143 & & \\
\hline [SIII] 9069 & 158 & & 170 & 2120: & 1895 & 78 & & & 6621 & 240 & & & & 433 & 572 \\
\hline [SIII] 9531 & 28 & & & 4599: & & 240 & & & & 617 & & & & 3333 & 1231 \\
\hline
\end{tabular}


PNG000.7-06.1 A little absorbed, inhomogeneous, bipolar PN presenting a sharp northern edge and a faint, extended western arc. Although no spectroscopic material was obtained for this interesting object having $\log F(\mathrm{H} \alpha)=-11.93 \mathrm{erg} \mathrm{cm}^{-2} \mathrm{~s}^{-1}$, its morphology suggests that it could be a Type-I PN (Torres-Peimbert \& Peimbert 1997 and references therein). Independently discovered by Beaulieu et al. (1999), who report $\log F(\mathrm{H} \alpha)=$ $-11.43 \mathrm{erg} \mathrm{cm}^{-2} \mathrm{~s}^{-1}$.

PNG001.3+06.3 An oblung, low excitation filamentary PN presenting large stratification effects, located at the edge of a heavily absorbed field. Being $\mathrm{H} \beta$ and $\lambda 5007 \AA$ of [OIII] quite faint in our spectrum, both $c(\mathrm{H} \beta)$ and the distance result uncertain.

PNG002.0+06.6 S-shape + elliptical disk in a very absorbed field. Very low excitation spectrum $(5007 \AA / \mathrm{H} \beta=0.25)$.

PNG002.2-01.2 An inhomogeneous, very absorbed, bipolar (butterfly? Type-I?)

PNG009.9+04.5 A lumpy, inhomogeneous, double ring presenting large stratification effects and a spectrum typical of Type-I PNe (Torres-Peimbert \& Peimbert 1997). Based on the detected $5755 \AA[\mathrm{NII}]$ auroral line, we derived an estimate of the electronic temperature from the [NII] $6584 / 5755$ ratio, $T$ e $=8900 \mathrm{~K}$. As argued in Sect. 4, the more plausible central star is candidate $b$

PNG011.2-02.7 A sharp, semi-circular arc in a heavily obscured field presenting a peculiar spectrum. The strong relative [SII] emissions indicate that the nebula is most likely shock excited. This is confirmed by the high [OII]/[OIII] ratio (Baldwin et al. 1981; Phillips \& Guzman 1998). Indeed in diagnostic diagrams (Tajitsu et al. 1999) its position is the same as that of Supernova Remnants! Because of these peculiarities the distance estimates reported in Table 2 are to be considered with care. In particular, the Balmer decrement in a shocked region is expected to be larger than the recombination value. This implies that the extinction based distance should be considered an upper limit. It is difficult to conciliate with the small angular size of PNG011.2-02.7, given the expected high velocity expansion rate of a $\mathrm{SN}$ remnant. We remind that remnant of the Kepler supernova is at a distance of $4.5 \mathrm{kpc}$ and has an angular radius of $1.5^{\prime}$. Being closer and significantly smaller, PNG011.2-02.7 would need to be much younger than the 400 yr old Kepler SNR.

One may also think to a ring nebula produced by the strong wind from some hot stars (which however does not appear on our frame). Unfortunately, the nebula is quite faint and our spectrum is of low $\mathrm{S} / \mathrm{N}$ and modest spectral resolution preventing any conclusion with respect to the nebular velocity field.

The present observational material makes the case for more detailed observations.

PNG011.7+00.2 A heavily absorbed bipolar, elliptical disk independently classified as a PN candidate by Ratag \& Pottasch (1991) \& Becker et al. (1994) on the basis of radio and infrared fluxes. It is the only IRAS source (IRAS 18073-1839) of the sample.

\section{Conclusions}

By direct inspection of the Palomar Observatory and ESO/SERC sky survey plates, we have discovered over a hundred candidates Galactic PNe. Here we report on the spectroscopic confirmation of 16 candidate PNe and one peculiar shocked nebula found in eight survey fields centered in the direction of the Galactic center. This increases roughly by $10 \%$ the number of known PNe in the area. We note that in the same fields we already identified other $17 \mathrm{PNe}$ candidates for which we still needs a spectroscopic confirmation.

The main nebular parameters and the temperature and luminosity of the central stars (when identified) have been derived.

The good agreement of the PN distances measured with two independent methods gives confidence in the conclusion that the new PNe are at intermediate evolutionary stage (cf. Schönberner 1981) and moderate distance, confined roughly to half the way to the Galactic Center. Hence this type of visual search appears to integrate the $\mathrm{PNe}$ candidates selected by the red-infrared comparison and radio searches, which are much more efficient for the compact, distant, heavily extincted objects (Van de Steene \& Jacoby 2001).

\section{References}

Acker, A., Ochsenbein, F., Stenholm, B., et al. 1992, Strasbourg-ESO Catalogue of Galactic Planetary Nebulae (ESO, Garching)

Aller, L. H. 1984, Physics of Thermal Gaseous Nebulae, ed. L. H. Aller, ISBN 90-277-1814-8, 1984 (Astrophysics \& Space Science Library), 112

Baldwin, J. A., Phillips, M. M., \& Terlevich, R. 1981, PASP, 93, 5

Beaulieu, S. F., Dopita, M. A., \& Freeman, K. C. 1999, ApJ, 515,610

Becker, R. H., White, R. L., Helfand, D. J., \& Zoonematkermani, S. 1994, ApJS, 91, 347

Boffi, F. R., \& Stanghellini, L. 1994, A\&A, 284, 248

Brocklehurst, M. 1971, MNRAS, 153, 471

Cappellaro, E., Benetti, S., Sabbadin, F., et al. 1994, MNRAS, 267,871

Cappellaro, E., Turatto, M., Sabbadin, F., \& Salvadori, L. 1990, A\&AS, 86, 503

Ciardullo, R., Jacoby, G. H., Feldmeier, J. J., \& Bartlett, R. E. 1998, ApJ, 492, 62

Cuisinier, F., Terzan, A., \& Acker, A. 1994, A\&A, 287, 227

Dopita, M. A., \& Meatheringham, S. J. 1990, ApJ, 357, 140

Durand, S., Acker, A., \& Zijlstra, A. 1998, A\&AS, 132, 13

Gleizes, F., Acker, A., \& Stenholm, B. 1989, A\&A, 222, 237

Gurzadyan, G. A. 1997, The Physics and Dynamics of Planetary Nebulae (Springer-Verlag, Berlin/Heidelberg)

Hamuy, M., Walker, A. R., Suntzeff, N. B., et al. 1992, PASP, 104,533

Hamuy, M., Suntzeff, N. B., Heathcote, S. R., et al. 1994, PASP, 106, 566

Jacoby, G. H. 1997, Planetary Nebulae, IAU Symp., 180, 448

Kerber, F., Lercher, G., \& Weinberger, R. 1996, A\&AS, 119, 423 
Kohoutek, L. 1994, Astronomische Nachrichten, 315, 235

Kistiakowsky, V., \& Helfand, D. J. 1995, AJ, 110, 2225

Kohoutek, L. 1997, Astronomische Nachrichten, 318, 35

Landolt, A. U. 1992, AJ, 104, 340

Lucke, P. B. 1978, A\&A, 64, 367

Osterbrock, D. E. 1989, Astrophysics of Gaseous Nebulae and Active Galactic Nuclei (University Science Books, Mill Valley, CA)

Phillips, J. P., \& Guzman, V. 1998, A\&AS, 130, 465

Pottasch, S. R. 1983, Planetary Nebulae, IAU Symp., 103, 391

Pottasch, S. R., Olling, R., Bignell, C., \& Zijlstra, A. A. 1988, A\&A, 205, 248

Pottasch, S. R. 1992, A\&AR, 4, 215

Pottasch, S. R. 1996, A\&A, 307, 561

Ratag, M. A., \& Pottasch, S. R. 1991, A\&AS, 91, 481

Sabbadin, F. 1986, A\&AS, 65, 301

Schönberner, D. 1981, A\&A, 103, 119
Seaton, M. J. 1979, MNRAS, 187, 785

Tajitsu, A., Tamura, S., Yadoumaru, Y., Weinberger, R., \& Köppen, J. 1999, PASP, 111, 1157

Torres-Peimbert, S., \& Peimbert, M. 1997, Planetary Nebulae, IAU Symp., 180, 175

Turatto, M., Cappellaro, E., Sabbadin, F., \& Salvadori, L. 1990, AJ, 99, 1170

Van de Steene, G. C. M., \& Pottasch, S. R. 1993, A\&A, 274, 895

Van de Steene, G. C., \& Jacoby, C. H. 2001, A\&A, 373, 536

Weinberger, R. 1977, A\&AS, 30, 343

Weinberger, R., Dengel, J., Hartl, H., \& Sabbadin, F. 1983, ApJ, 265, 249

Wyatt, S. P. 1978, Planetary Nebulae, IAU Symp., 76, XVII

Zanin, C., Cappellaro, E., Sabbadin, F., \& Turatto, M. 1997, Planetary Nebulae, IAU Symp., 180, 26 